\title{
Effects of Display Layout on Gaze Activity During Visual Search
}

\author{
Jérôme Simonin, Suzanne Kieffer, and Noëlle Carbonell \\ LORIA, Campus Scientifique, BP 239, F54506, Vandœuvre-lès-Nancy Cedex France \\ \{Jerome.Simonin, Suzanne.Kieffer, Noelle.Carbonell\}@loria.fr
}

\begin{abstract}
We report an experimental study that aims at investigating the influence of spatial layout on visual search efficiency and comfort. 4 layouts were used for displaying 120 scenes comprising 30 realistic colour photos each: random, elliptic, radial and matrix-like. Scenes (30 per structure) were presented to 5 participants who had to select a pre-viewed photo in each scene using the mouse. Eye-tracking data indicate that elliptic layouts provided better visual comfort than any of the other layouts (shortest scan paths), and proved to be more efficient than matrix layouts (shorter search times). These results are statistically significant (paired t-tests).
\end{abstract}

\section{Introduction}

Entertainment and commercial Web-sites, information kiosks and public terminals tend to display a growing number of pictures simultaneously: video and movie stills, CD sleeves, book covers, etc. Personal electronic archives and directories are increasingly cluttered with collections of photos, scanned documents, videos. It is a standard practice for designers of image browsers, to display information items in the form of 2D arrays that users browse through, using horizontal and vertical scrollbars. Current products (e.g., ACDSee, PhotoSuite or ThumbsPlus) make general use of scrollable $2 \mathrm{D}$ arrays of file icons or miniatures for displaying folder contents. Research prototypes of multimedia news summaries [5] or "zoomable" image browsers [1] also use 2D array presentations exclusively. Designers and researchers seem to take it for granted that 2D arrays are more efficient and comfortable than any other structure for presenting picture sets.

However, the prevalent use of 2D array layouts for displaying collections of images has yet to be grounded on established ergonomic criteria. Empirical and experimental studies are needed to determine the actual efficiency and visual comfort of possible display layouts. To our knowledge, this ergonomic issue has not yet been addressed. Published research on the usability of picture browsers amounts to a few studies meant to assess the overall ergonomic quality of specific products [3] or research prototypes, for instance Shoebox [6] and PhotoMesa [4]. The aim of the experimental study presented here is to obtain a meaningful insight into the actual influence of display layout on visual search performance and comfort, for picture sets.

Experimental design and set-up, which take advantage of the conclusions of an earlier pilot study [2], are first described. Then, quantitative and qualitative results are presented and discussed. The paper ends with a summary of conclusions stemming from these results together with a brief sketch of future research directions. 


\section{Experimental Design and Set-Up}

Five experienced computer users with ages between 24 and 29 and normal sight (Bioptor test kit) carried out 120 visual search tasks in scenes displayed on a 21 ' screen (1280 x 1024 pixel resolution). Each scene included 30 realistic colour photos arranged along four different symmetrical structures (see figure 1): Matrix-like (2D array), Elliptic (two concentric ellipses), Radial (eight radii along medians and diagonals of the screen), and Random (i.e., random placing). Elliptic and Radial structures were meant to schematise information presentation layouts often used in everyday life (e.g., dials).

For each scene, participants had to locate a pre-viewed photo in the scene, and to select it as fast as they could using the mouse. The isolated target was first displayed in the centre of the screen during three seconds; then participants clicked on a button in the centre of the screen for launching the scene display; thus, mouse initial position was identical for all search tasks. Participants' gaze activity was recorded using a head-mounted eye-tracker (ASL). After a short presentation followed by a calibration stage ( 5 min.), participants carried out 6 trial tasks, then the 120 experimental tasks (30 per structure).

3600 photos pulled from popular Web sites were sorted out into 40 themes or so (e.g., sports, animals, monuments) and sub-themes. Each scene was made up of photos belonging to the same theme or sub-theme in order to reduce intra-scene variability in visual saliency and subjective appeal; thus, "pop out" effects that might interfere with the possible influence of display layout on gaze activity were avoided. For this reason also, each target was chosen among photos with medium visual saliency in the scene. Photos were placed randomly in the scenes save for targets which were placed manually in all possible locations in the 120 scenes; thus, possible effects of target
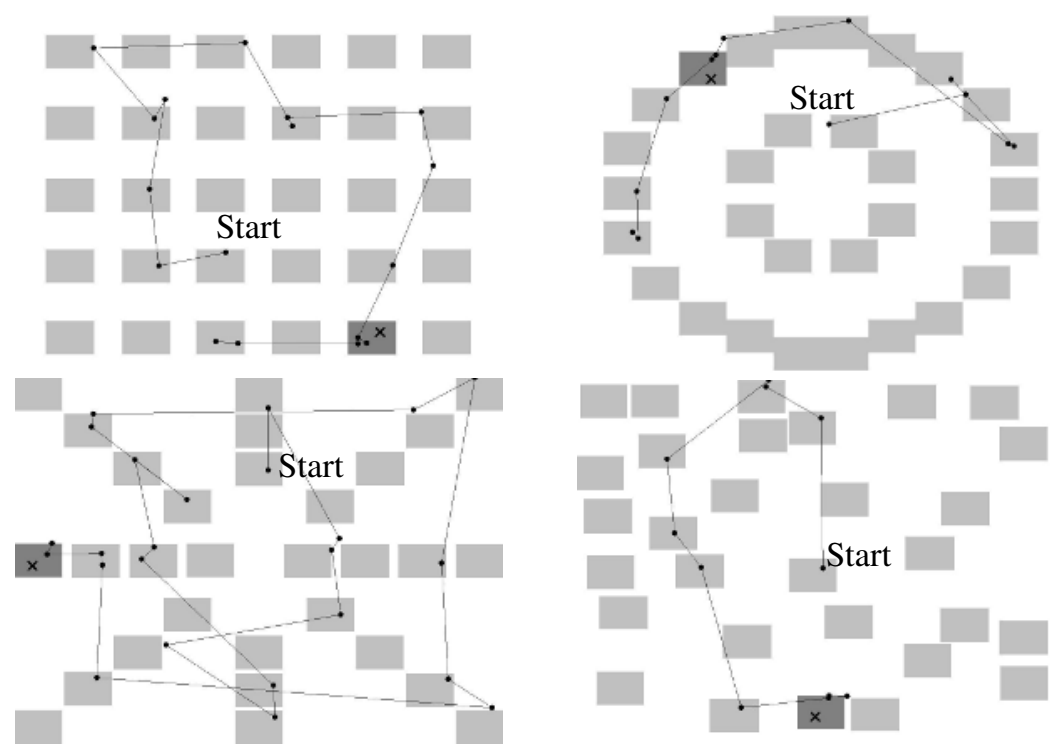

Fig. 1. Standard scan paths for each spatial structure. The target is represented by a dark gray box, the selection click by a cross, fixations by black dots. 
position on visual search efficiency were prevented. Scenes were ordered randomly, regardless of their structure, and a different order was assigned to each participant so as to neutralize possible sequence and task learning effects.

Display layout was the main independent variable. Overall task efficiency and effectiveness were assessed using target selection time and task failure rate as dependent variables. To evaluate visual search efficiency and comfort, five measures were computed over the time interval (st) ranging from scene display onset to first fixation on the target offset: duration of st (D_st), number and duration of fixations ( $\mathrm{Nf}$ and Df), saccade duration (Ds), scan path length (Lsp). All these measures are useful for assessing visual search efficiency; some of them also contribute to evaluating visual comfort, since longer search times, higher fixation numbers and longer scan paths imply higher gaze activity, hence greater visual fatigue.

Results are presented in the next section. We discuss their contribution to validating the two following working hypotheses which are based on the general assumption that display structure influences eye movements during visual search. Given our visual material, we expect worse performances for unstructured displays (c.f. the Random structure) than for any structured display since, if spatial structure influences scan paths it will favour thorough systematic search and limit useless backtracking (hypothesis 1). In addition, best performance results will be obtained for Elliptic layouts which have a greater influence on gaze trajectories than Matrix layouts and do not favour backtracking like the Radial structure; worst results are expected for $M a$ trix layouts which influence scan paths least (hypothesis 2).

\section{Results: Presentation and Discussion}

Participants' task performances do not confirm our working hypotheses. Task failures had to be left out, due to their small number (45 for 600 tasks) and high interindividual differences (from $2.5 \%$ to $11.7 \%$ of 120 tasks). Comparisons between target selection times by structure do not reach statistical significance (paired t-tests), averages ranging from $4.17 \mathrm{sec}$. (Elliptic structure) to $4.53 \mathrm{sec}$. (Matrix structure). To explain these results, it may be put forth that, as selection time includes search time for the target (about $2.6 \mathrm{sec}$.) and mouse move-and-click duration (over $1.6 \mathrm{sec}$.), the effects of spatial structure on search time might go unnoticed.

Table 1. Averaged eye-tracking measures per structure during target search (st), 5 participants: duration of st, number of fixations, fixation and saccade duration, scan path length

\begin{tabular}{lccccc}
\hline $\begin{array}{c}\text { Variables } \\
\text { Number of values }\end{array}$ & $\begin{array}{c}\text { Random } \\
141\end{array}$ & $\begin{array}{c}\text { Ellipse } \\
143\end{array}$ & $\begin{array}{c}\text { Matrix } \\
142\end{array}$ & $\begin{array}{c}\text { Radial } \\
141\end{array}$ & Results of paired t-tests \\
\hline D_st (sec.) & 2.48 & 2.27 & 2.89 & 2.68 & E-M: t=-2; $\mathrm{p}=0.0462$ \\
\hline Nf & 9.38 & 8.75 & 10.78 & 9.76 & not significant \\
\hline Df (ms.) & 153 & 153 & 157 & 157 & not significant \\
\hline Ds (ms.) & 134 & 130 & 129 & 143 & not significant \\
\hline Lsp (pixels) & 1818 & 1440 & 1951 & 1973 & see footnote $^{1}$ \\
\hline
\end{tabular}

${ }^{1}$ Rand.-E : $\mathrm{t}=2.08 ; \mathrm{p}=0.0380 / \mathrm{M}-\mathrm{E}: \mathrm{t}=2.53 ; \mathrm{p}=0.0119$ / Rad. $\mathrm{E}: \mathrm{t}=2.44 ; \mathrm{p}=0.0462$ 
Eye-tracking data (see table 1) support this interpretation. Target localization time is significantly shorter for Elliptic layouts than for Matrix layouts. In addition, scan path length is significantly smaller for Elliptic layouts compared to the three other structures. These results validate hypothesis 2 partly, while they contradict hypothesis 1. Qualitative analyses of participants' gaze activity during target search help to interpret them (see figure 1). Scan paths seem to have been more influenced by Radial and Elliptic layouts than by Matrix layouts: only $17 \%$ of saccades or so are jumps from one ellipse to the other or from one radius to a non neighbouring one, whereas saccade directions are more varied for Matrix layouts (34\% of saccades follow lines, $28 \%$ diagonals, and $23 \%$ columns) with higher inter-individual differences (e.g., from $21 \%$ to $35 \%$ for moves along diagonals). Besides, two strategies were used for exploring Radial structures, one with few backtrackings (2 participants), the other with many (see figure 1 ). These observations explain why hypothesis 2 was only partly confirmed by quantitative results. As for hypothesis 1 , scan path analysis shows that participants moved from one "cluster" of photos to another in a Random structured scene, suggesting that Random layouts cannot be viewed as unstructured layouts.

\section{Conclusion}

We performed an experimental study that aimed at investigating the influence of spatial layout on visual search efficiency and comfort. 4 layouts were used for displaying sets of 30 realistic colour photos: random, elliptic, radial and matrix-like. 120 scenes (30 per structure) were presented to 5 participants who had to select a previewed photo in each scene using the mouse. Eye-tracking data analyses indicate that Elliptic layouts provided better visual comfort than any of the other layouts (shortest scan paths), and proved to be more efficient than Matrix layouts (shorter search times). These results are significant (paired t-tests). They may be useful for improving the design of photo visualization and browsing. Future work will focus first on modelling user gaze strategies in order to refine the comparison between Radial and Elliptic layouts, then on testing whether similar results are obtained for displays of sensibly larger collections of visual items.

\section{References}

1. Bederson, B.B. (2001). PhotoMesa: a Zoomable Image Browser Using Quantum Treemaps and Bubblemaps. Proc. $14^{\text {th }}$ ACM Annual Symposium on User Interfaces Software and Technology (UIST'01), CHI Letters, 3(2), 71-80.

2. Carbonell, N., Kieffer, S. (2005). Do oral messages help visual exploration?", in J. van Kuppevelt, L. Dybkjaer, N. Bernsen (Eds.), Advances in Natural, Multimodal Dialogue Systems, Springer (Boston: Kluwer Inc.), 27 pp. (to appear).

3. Combs, T.T., Bederson, B.B. (1999). Does Zooming Improve Image Browsing. Proc. ACM Conference on Digital Liraries (DL'99), ACM Press, New York, pp. 130-137.

4. Khella, A., Bederson, B. (2004). Pocket PhotoMesa: a Zooming Image Browser for the Pocket PC. Technical Report, University of Maryland HCI Lab, 9 pp.

5. Maybury, M. (2000). News on demand. Communications of the ACM, 43(2), 32-34.

6. Shneiderman, B., Kang, H., Kules, B., Plaisant, C., Rose, A., Rucheir, R. (2002). A Photo History of SIGCHI: Evolution of design from personal to public. Interactions, 9(3), 17-23. 